(C) 2017 IEEE. Personal use of this material is permitted. Permission from IEEE must be obtained for all other uses, in any current or future media, including reprinting/republishing this material for advertising or promotional purposes, creating new collective works, for resale or redistribution to servers or lists, or reuse of any copyrighted component of this work in other works. 


\title{
A Generalized Continuous Wave Synthetic Aperture Radar
}

\author{
Yijiang Nan, Xiaojing Huang and Y. Jay Guo \\ University of Technology Sydney \\ Sydney, Australia \\ Emails: Yijiang.Nan@student.uts.edu.au, \{Xiaojing.Huang, Jay.Guo\}@uts.edu.au
}

\begin{abstract}
Attention has been devoted to Synthetic Aperture Radar (SAR) for half a century. Though it is a well-proven remote sensing technique, conventional pulsed SAR has several inherent limitations. In this paper, we present a new SAR concept, called Generalized Continuous Wave SAR (GCW-SAR). By using continuous wave signaling, the GCW-SAR system achieves better performance and overcomes the limitations such as the minimum antenna area in conventional SAR. Unlike the frequency modulated continuous wave SAR (FMCW-SAR) system, the GCWSAR image is reconstructed by correlation between the sampled raw data and the location dependent reference signals. A fast image reconstruction algorithm is also presented in the paper. The principle of GCW-SAR and the effectiveness of the proposed algorithm are validated by numerical simulation results.
\end{abstract}

Index Terms-Continuous Wave, Correlation, Image Reconstruction, Synthetic Aperture Radar.

\section{INTRODUCTION}

Synthetic Aperture Radar (SAR) is a well-proven powerful remote sensing technique that can obtain high-resolution images by using transmitted wide band waveforms and exploiting the relative motion between platform and targets. However, the conventional SAR systems make use of extremely low duty cycle coherent pulses to obtain both range and azimuth resolutions. Such pulsed radar systems usually have to transmit waveforms at a high power level and thus are of high cost and less compact. Therefore, they are hardly applicable to lowcost civil instruments such as unmanned aerial vehicle (UAV) or small aircraft solution [1]. On the other hand, the singleantenna SAR systems cannot achieve high azimuth resolution and wide swath simultaneously. The tradeoff among many of the SAR system parameters results in a minimum antenna area constraint [2].

To overcome the above fundamental limitations, several new SAR models and novel algorithms have been proposed in recent years. The frequency modulated continuous wave (FMCW) SAR system [1], [3] operates with a constant low transmission power and is of great interest to the civil market and military applications, but nonlinearities in its beat signal deteriorate its performance at a large distance and its limited power also results in a short-distance imaging. Multiple-input and multiple-output synthetic aperture radar (MIMO-SAR) [4]-[6] is also an attractive concept, which employs digital beamforming (DBF) [4] techniques to improve the imaging. Unfortunately, the better performance results from the MIMO

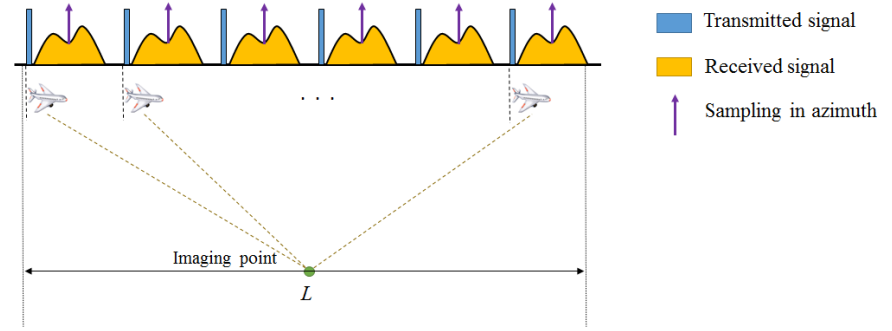

(a)

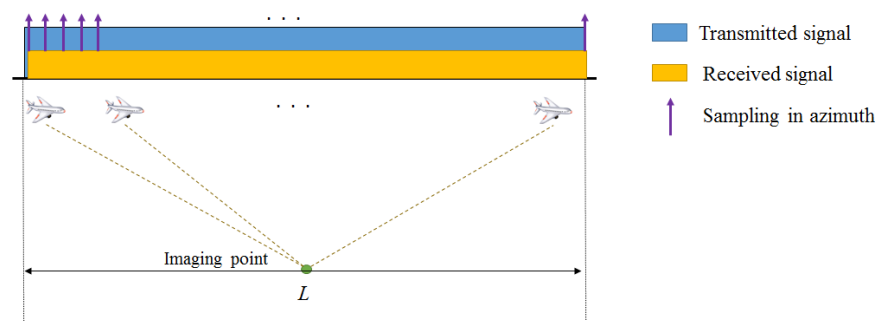

(b)

Fig. 1. Different transmit and receive models in SAR (a) and GCW-SAR (b), where $L$ is the beamwidth in azimuth.

characteristics and thus the MIMO-SAR systems cannot break the tradeoff between azimuth resolution and range ambiguity in conventional SAR systems.

This paper proposes a new concept for future high performance remote sensing, named Generalized Continuous Wave SAR (GCW-SAR), which combines continuous wave (CW) radar with full-duplex radio technique [7]. In a full-duplex system, transmission and reception are conducted at the same time in the same band and the received signal can be extracted after self-interference cancellation. Applying this technique to the SAR system, the GCW-SAR system transmits a continuous wave signal and receives its echoes simultaneously. As is seen from Fig .1, in the SAR system, the length of received signal during each pulse repetition interval (PRI) is proportional to the range swath. A wider swath may result in a lower sampling rate in azimuth. However, in the GCW-SAR system, both the azimuth and range samplings are made from the continuously received signal and the azimuth sampling rate is therefore independent of the range swath. Assuming the same radar sensitivity, the new system will offer a number of advantages, which are further explained in details as follows. 
1) Low power operation: The GCW-SAR system uses less signal power than conventional SAR for imaging from the same distance. In conventional SAR, a frequency modulated pulse (called chirp signal) is emitted at the start of each PRI and echoes reflected back from the scatters on the ground are then stored on-board. Due to the on/off switching between transmit and receive, the low duty cycle PRI leads to less signal energy received by SAR antenna. With continuous wave signaling, the GCW-SAR transmits signal in full duty cycle and receives more reflected signal energy, which can improve the signal-to-noise ratio (SNR) with the same transmit power or reduce the transmit power of the system to achieve the same SNR.

2) No limitation of minimum antenna area: The GCWSAR system breaks the limitation of minimum antenna area in pulsed SAR, and obtains both high azimuth resolution and wide range swath. The received signals in GCW-SAR system provide the reflected information of the scatters at any time when the scatters are illuminated by the radar transmitted signal beam. Therefore, there will be no slow time involved in GCW-SAR and the azimuth resolution and swath will be no more restricted.

3) Wider application than FMCW SAR: The FMCW SAR transmits periodic linearly frequency modulated signal and mixes the received signal down to baseband with the transmitted signal (called deramp on receive) [1]. The beat signal is obtained via low-pass filtering of the deramped baseband signal and the ranging is obtained by frequency offset analysis of the beat signal. However, the FMCW SAR is still restricted by the minimum antenna area and its performance is poor for long-range application due to the nonlinearity in its beat signal. Actually, the FMCW SAR is only a special case of the GCW-SAR system, which limits the transmitted waveform to frequency modulated continuous wave and only considers the frequency difference between the echo signal and the local reference signal for ranging. After self-interference cancellation, the GCWSAR system can receive the reflected signals continuously and reconstruct the target image through signal correlation processing with the target location dependent reference signal. Therefore, the new system is suitable for both short and long range applications.

4) Flexibility in signal design and system configuration: Firstly, the transmitted waveform design in GCW-SAR is much more flexible. The new system is allowed to transmit a signal with any waveform such as a periodic chirp signal with large bandwidth or just a single-frequency signal. Secondly, the GCW-SAR system can use either a single antenna or a multiple-input multiple-output antenna array. Therefore, there are many GCW-SAR modes to meet different sensing requirements. For instance, if the system transmits a wide band signal, a three-dimensional image can be reconstructed with multiple receivers. A singlefrequency transmitted signal with linear antenna array can look down the Earth on both sides of the radar platform and provide much wider swath.
Some technical challenges may exist in the implementation of the GCW-SAR. Firstly, transmitter self-interference needs to be solved before the GCW-SAR becomes a reality. Secondly, image reconstruction may require complicated signal processing since the range and azimuth informations are mingled with the GCW-SAR raw data. Regarding the first issue, a number of self-interference cancellation techniques have been proposed in recent years, and the increasingly better suppression of transmitted signal will bring the GCW-SAR into practical operation. Therefore, this paper will only focus on the second challenging issue in the GCW-SAR system.

As we all know, SAR signal processing, including various time and/or frequency domain imaging algorithms using fast Fourier transform (FFT), is essentially a signal correlation between the received raw data and a reference signal with different time delay and different Doppler frequency shift. Thus, in this paper, we first consider the GCW-SAR signal processing as a signal correlation or matched filtering process. Then, we propose a fast GCW-SAR imaging algorithm, named Piecewise Constant Doppler (PCD) Algorithm, which can reduce the GCW-SAR signal processing complexity significantly. For simplicity, this paper only describes the stripmap GCW-SAR (single-antenna, side-looking and constant antenna pointing direction). The other GCW-SAR modes will be studied in our future work.

The remaining sections of this paper are organized as follows. The GCW-SAR principle is described in Section II. Section III derives the PCD algorithm for GCW-SAR and the numerical simulations are shown in Section IV. Finally, Section $\mathrm{V}$ concludes this paper with a short discussion.

\section{GCW-SAR PRINCIPLE}

\section{A. Imaging in pulsed SAR}

Following the stop-and-go approximation [8], the raw data in conventional SAR can be represented as a two-dimensional data matrix. The first dimension corresponds to the range direction (fast time). The SAR transmits a pulse whenever it travels for a pulse repetition time with a constant speed, thus forming the second dimension of the data matrix known as azimuth direction (slow time). After the range compression, the reflected signals from different distances in range direction are separated. By collecting the compressed signal with the same range distance and making azimuth compression, a focused image is finally formed from the SAR raw data.

\section{B. Generalized imaging in $G C W-S A R$}

In GCW-SAR system, most of the current SAR algorithms are no longer valid. Different from the two-dimensional raw data acquisition in SAR, the raw data in GCW-SAR are onedimensional discrete values sampled from the continuously received signal. Obviously, there is no slow time in the GCW-SAR imaging algorithm and hence no explicit data compression from either range or azimuth direnction. In this section, the GCW-SAR geometry is presented first and then the generalized imaging algorithm is proposed. 


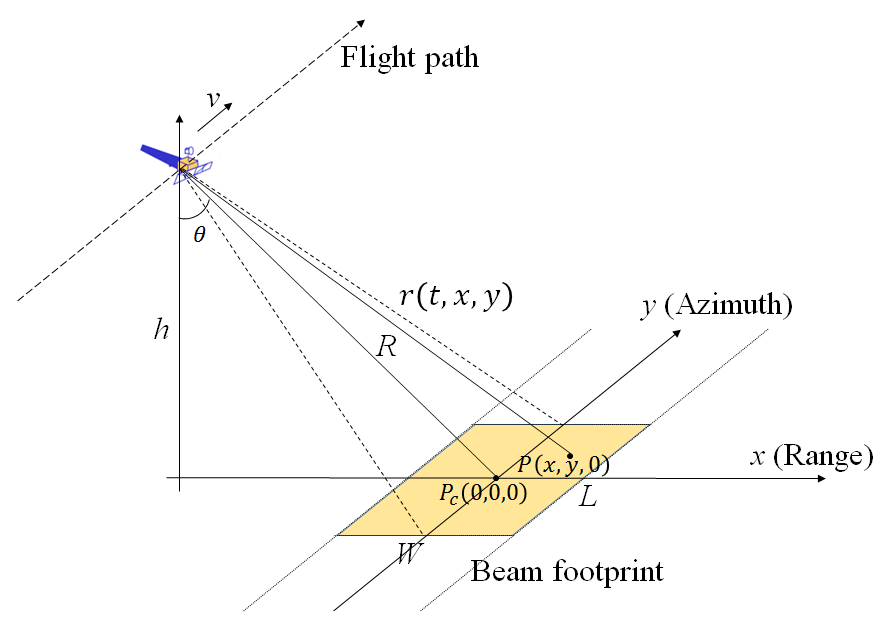

Fig. 2. GCW-SAR geometry.

1) GCW-SAR geometry: The GCW-SAR geometry is considered in the Cartesian coordinate system, as is shown in Fig .2. The radar, at a height $h$, travels with a constant speed $v$ and its flight path is in parallel to the $y$-axis. The illuminated target area (beam footprint) is illustrated as the shaded area, where the center point $P_{c}$ is set to the origin $(0,0,0)$. W and $L$ are the range and azimuth widths of the beam footprint respectively and the beam incident angle is set to $\theta$. The shortest distance from $P_{c}$ to the radar is $R$. The distance describing the path of signal propagation from the radar to an arbitrary point $P$ in the beam footprint is

$$
r(t, x, y)=\sqrt{(R \sin \theta+x)^{2}+(y-v t)^{2}+h^{2}}
$$

where $t \in(-T / 2, T / 2) . T$ is the traveling time over the synthetic aperture, which is equal to $L / v$.

2) Generalized imaging algorithm: According to the pulse compression theory, the imaging reconstruction is generally a cross-correlation between received signal and a reference signal. After the round-trip propagation time from the transmit antenna to the target area, the demodulated received signal can be written as (2) on top of next page, where $\sigma(x, y)$ is the radar cross section (RCS) of point $(x, y)$, the center of beam footprint is $(0, v t), s(t)$ is the baseband transmitted signal, $c$ is the light speed, and $\lambda$ is the wavelength of the transmit beam. Compared with the transmitted signal, the received signal actually varies in two aspects: the first one is the time delay and the second one is the relative Doppler frequency shift.

Following the stop-and-go model, the conventional SAR system transmits the same chirp signal pulse at the beginning of each PRI. During each PRI, the Doppler shift can be considered as a constant and the signal delay in fast time represents the range of the target. Thus, the reference signal is set to the received signal from the center point $(0,0)$ and the image of a target can be reconstructed by performing range and Doppler compressions separately in fast time and slow time domains.
In GCW-SAR system, however, the Doppler frequency shift is hidden in the one-dimensional raw data and we cannot separate the fast and slow times. Fortunately, the time delay and Doppler frequency shift still exist in the echoes so that the RCS can be retrieved by correlating the received raw data with location dependent reference signals. For an arbitrary point $\left(x_{i}, y_{i}\right)$, the reconstructed image is actually the integral of the ambiguity function [9] from point $\left(x_{i}, y_{i}\right)$, as is derived in (3) and (4) on top of next page where the ambiguity function is defined as a two-dimensional function of distance in $x$-axis and $y$-axis showing a distorted image of a point scatter. Assuming that the bandwidth of the transmitted signal is $B$ and the width of the antenna aperture is set to $L_{a}$, the range and azimuth resolutions in generalized SAR system are derived as

$$
\tau_{x}=c / 2 B \sin \theta
$$

and

$$
\tau_{y}=L_{a} / 2
$$

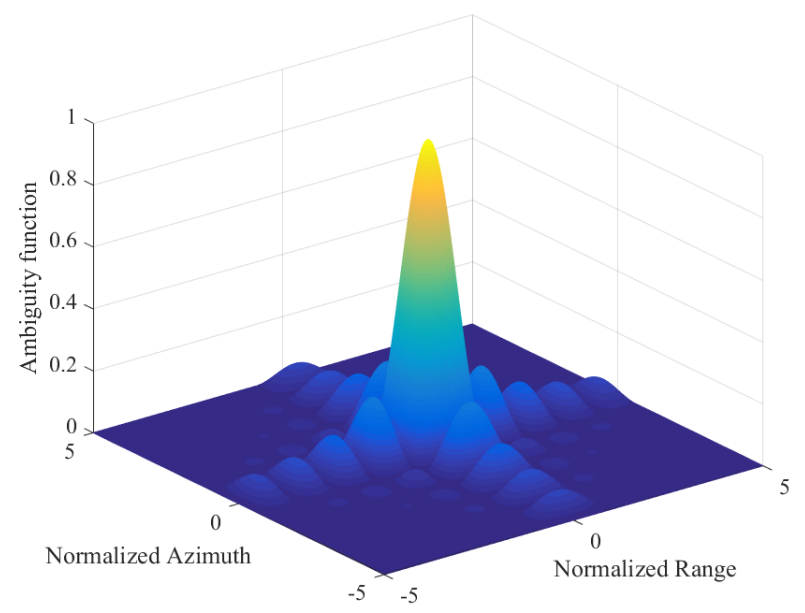

Fig. 3. GCW-SAR ambiguity function.

After the $x$-axis and $y$-axis are normalized by the range and azimuth resolutions respectively, an example of GCWSAR ambiguity function is shown in Fig. 3. Obviously, the imaging process approximately approaches the integral of the ambiguity function in the mainlobe, and thus can be implemented as follows.

Assume that the beam footprint is filled with a set of uniformly distributed point scatters with spacings along $x$ and $y$ axises equal to the range and azimuth resolutions respectively. The expected received signals from these scatters are used as the reference signals. After performing correlation between the actually received signals and the reference signals, the GCW-SAR finally reconstructs a two-dimensional image.

\section{Piecewise Constant Doppler Algorithm}

With the generalized imaging algorithm, a reference signal is necessary for each pixel in the GCW-SAR image. In order 


$$
\begin{gathered}
s_{r}(t)=\int_{-\frac{W}{2}}^{\frac{W}{2}} \int_{v t-\frac{L}{2}}^{v t+\frac{L}{2}} \sigma(x, y) s\left(t-\frac{2 r(t, x, y)}{c}\right) e^{-j \frac{4 \pi}{\lambda} r(t, x, y)} d x d y \\
\int_{\frac{y_{i}}{v}-\frac{T}{2}}^{\frac{y_{i}}{v}+\frac{T}{2}} s_{r}(t) s^{*}\left(t-\frac{2 r\left(t, x_{i}, y_{i}\right)}{c}\right) e^{j \frac{4 \pi}{\lambda} r\left(t, x_{i}, y_{i}\right)} d t=\int_{-\frac{W}{2}}^{\frac{W}{2}} \int_{v t-\frac{L}{2}}^{v t+\frac{L}{2}} \sigma(x, y) A_{x_{i}, y_{i}}(x, y) d x d y \\
A_{x_{i}, y_{i}}(x, y)=\int_{\frac{y_{i}}{v}-\frac{T}{2}}^{\frac{y_{i}}{v}+\frac{T}{2}} s\left(t-\frac{2 r(t, x, y)}{c}\right) s^{*}\left(t-\frac{2 r\left(t, x_{i}, y_{i}\right)}{c}\right) e^{j \frac{4 \pi}{\lambda}\left(r\left(t, x_{i}, y_{i}\right)-r(t, x, y)\right)} d t
\end{gathered}
$$

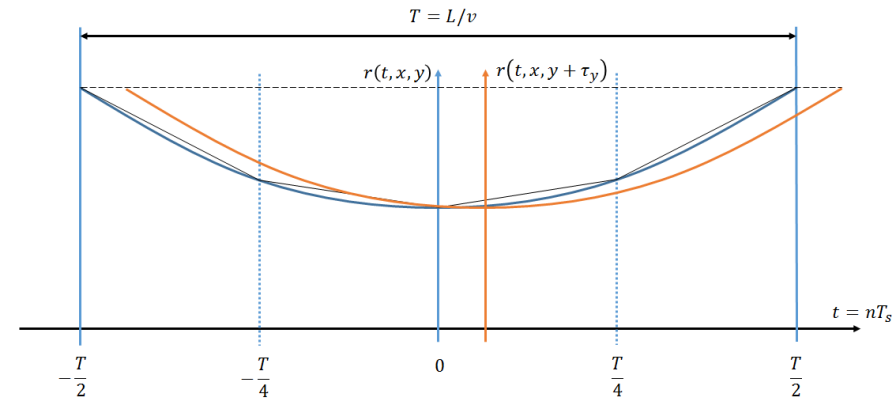

Fig. 4. The variation of distance between an imaging point to the radar.

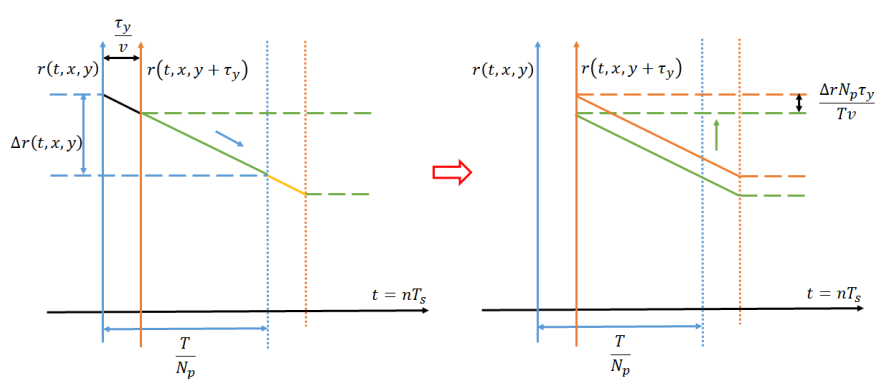

Fig. 5. The updating process for each line segment.

to obtain a high-resolution and wide-swath image, the number of reference signals is significant, which leads to great signal processing complexity. Assume that the number of image points is set to $N \times M$, where $N=L / \tau_{x}$ and $M=W / \tau_{y}$. For each point, the system performs a correlation operation using the received raw data and the corresponding stored reference signal. Thus, the complexity of the generalized imaging algoritm in GCW-SAR is approximately equal to $\mathcal{O}\left(N \times M \times N_{T}\right)$, where $N_{T}$ is the number of complex multiplications for each correlation operation. Aimed at reducing the complexity and the number of data storage, we propose a fast imaging algorithm named Piecewise Constant Doppler Algorithm, which can improve the efficiency of the GCW-SAR signal processing.

The received signal from an arbitray point $(x, y)$ changes continuously in response to its distance $r(t, x, y)$ to the radar. Assuming that the platform is moving at a constant speed in a straight line, the variation of the distance is equal to a parabola. In order to deal with the non-linear variation of $r(t, x, y)$, the parabola is divided into a number of segments linked end to end and the variation in each segment is approximately linear. The number of linear segments is set to $N_{p}$.

With the assistance of the linear approximation, the correlation operation can be changed to a recursive updating process. As shown in Fig. 4 for the case of $N_{p}=4$, the distance $r\left(t, x, y+\tau_{y}\right)$ for a neighboring point $\left(x, y+\tau_{y}\right)$ is actually a parallel shift of $r(t, x, y)$. Within each segment, all signal samples incur the same time delay and Doppler frequency shift because of the linear approximation. Therefore, correlation result for point $\left(x, y+\tau_{y}\right)$ can be updated from the previous one. Fig. 5 shows the updating process for each segment and the details are further explained as follows:

1) Due to the shift of traveling time slot, the first step in the updating process is to update the integration intervel of the correlation operation from $\left(-\frac{T}{2}+\frac{T}{N_{p}}\left(n_{p}-1\right),-\frac{T}{2}+\frac{T}{N_{p}} n_{p}\right)$ to $\left(-\frac{T}{2}+\frac{T}{N_{p}}\left(n_{p}-1\right)+\frac{\tau_{y}}{v},-\frac{T}{2}+\frac{T}{N_{p}} n_{p}+\frac{\tau_{y}}{v}\right)$, where $n_{p}$ is the index of a linear segment. From the left part of Fig. 5 , it is easily seen that the interval indicated in black is shifted out and the interval indicated in yellow is shifted in whereas the interval indicated in green remains unchanged.

2) The difference between $r\left(t, x, y+\tau_{y}\right)$ and $r(t, x, y)$ is a constant in each linear segment corresponding to a constant Doppler frequency shift. The second step is to compensate the Doppler frequency shift in the baseband transmitted signal which is seen as from the right part of Fig. 5. Since $\frac{2\left(r\left(t, x, y+\tau_{y}\right)-r(t, x, y)\right)}{c}$ is very small, the time delay can be neglected and the correlation result for point $\left(x, y+\tau_{y}\right)$ can be derived after multiplying the correlation result for point $(x, y)$ by $e^{-j \frac{4 \pi}{\lambda} \frac{\Delta r N_{p} \tau_{y}}{T v}}$, where $\Delta r$ is equal to $r\left(-\frac{T}{2}+\frac{T}{N_{p}} n_{p}, x, y\right)-r\left(-\frac{T}{2}+\frac{T}{N_{p}}\left(n_{p}-1\right), x, y\right)$.

The reconstructed image for point $\left(x, y+\tau_{y}\right)$ can be obtained by summing up the correlation results for all $N_{p}$ segments. We see that instead of performing correlation operation for each pixel individually, the PCD algorithm updates the correlation result recursively and thus saves much data storage and reduces the computational complexity.

\section{Simulation RESUlts}

In order to validate the GCW-SAR principle, a 4-point target imaging process is designed and simulated. For the sake of comparison, the coordinate $x$ and $y$ are normalized by the range and azimuth resolutions $\tau_{x}$ and $\tau_{y}$ respectively. Assume that there are 4 targets on the ground, where point $\mathrm{A}$ is the 
coordinate origin $(0,0)$ and the other three points B, C, D are located at $(-2,0),(0,6),(-2,6)$ respectively. The received signal starts to be sampled when the origin enters the beam footprint.

Fig. 6 shows the reconstructed image of the GCW-SAR. The received energy of different points has been separated and focused on the corresponding imaging points respectively. Assuming that the targets have the same RCS and the same fading channel state, Fig. 6 shows that the reconstructed image of GCW-SAR can approach the highest resolution.

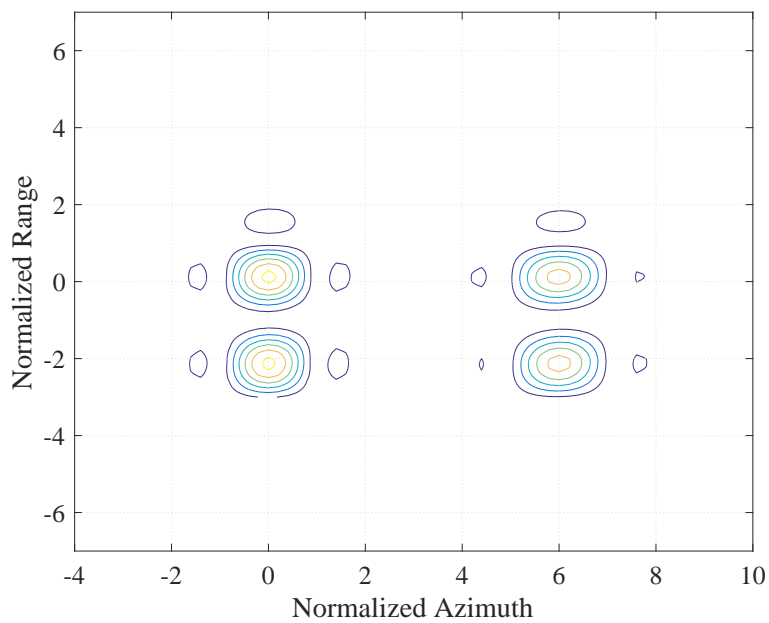

Fig. 6. Reconstructed image of the GCW-SAR.

The comparison of performance between GCW-SAR and SAR can be seen from the Fig. 7. With the same parameters, the Doppler sampling rate of the GCW-SAR is not limited by the minimum antenna area and the system acquires more reflected information. Hence, it can reconstruct a sharper image and achiveve higher resolution.

\section{CONCLUSION}

In this paper, a new SAR technique, called Generalized Continuous Wave SAR, is introduced and the corresponding fast imaging algorithm is proposed. By using the transmitted continuous wave, the GCW-SAR system can acquire more reflected information and reconstruct better image than the conventional SAR. Continuous wave signaling reduces the transmitted signal power and/or improves the SNR. It also removes the minimum antenna area constraint in the pulsed SAR. Simulation results show that GCW-SAR is an attractive technique which performs better than conventional SAR.

The proposed GCW-SAR concept can be also combined with the multiple-input and multiple-output radar to further improve the system performance. In our future work, we will continue the research and develop better algorithms for GCW$\mathrm{SAR}$ in various operation modes.

\section{REFERENCES}

[1] A. Meta, P. Hoogeboom, and L. P. Ligthart, "Signal Processing for FMCW SAR," IEEE Transactions on Geoscience and Remote Sensing, vol. 45, no. 11, pp. 3519-3532, Nov 2007.

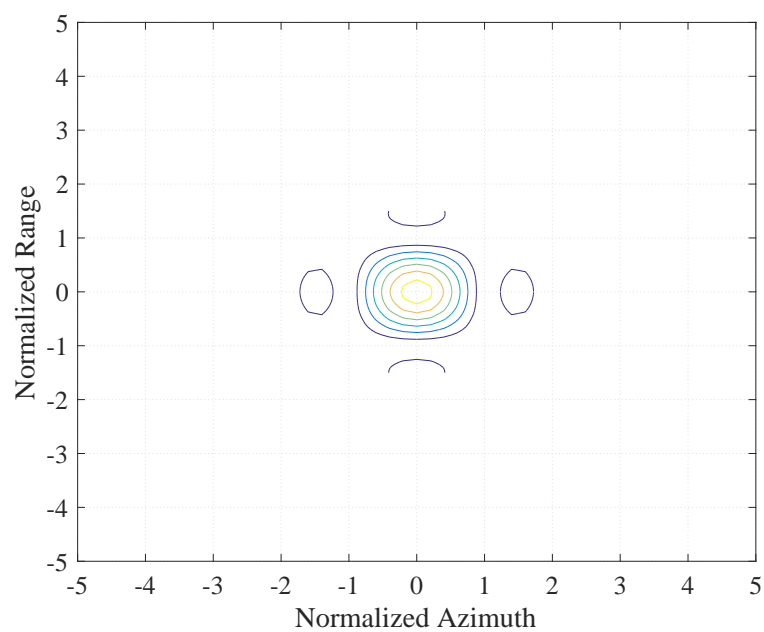

(a)

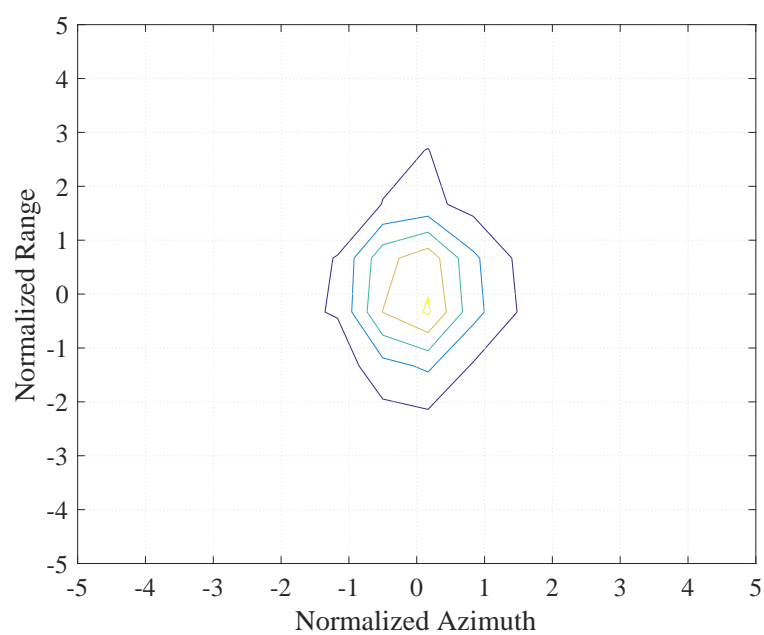

(b)

Fig. 7. Comparison between GCW-SAR and SAR.

[2] A. Currie and M. A. Brown, "Wide-swath SAR," IEE Proceedings F Radar and Signal Processing, vol. 139, no. 2, pp. 122-135, April 1992.

[3] A. Meta, P. Hoogeboom, and L. Ligthart, "Range Frequency Nonlinearity Correction for FMCW SAR," European Patent Application, no. $06076057.6,2006$

[4] G. Krieger, N. Gebert, and A. Moreira, "Multidimensional Waveform Encoding: A New Digital Beamforming Technique for Synthetic Aperture Radar Remote Sensing," IEEE Transactions on Geoscience and Remote Sensing, vol. 46, no. 1, pp. 31-46, Jan 2008.

[5] J. H. Kim, M. Younis, A. Moreira, and W. Wiesbeck, "A Novel OFDM Chirp Waveform Scheme for Use of Multiple Transmitters in SAR," IEEE Geoscience and Remote Sensing Letters, vol. 10, no. 3, pp. 568-572, May 2013.

[6] G. Krieger, "MIMO-SAR: Opportunities and Pitfalls," IEEE Transactions on Geoscience and Remote Sensing, vol. 52, no. 5, pp. 2628-2645, May 2014.

[7] S. Hong, J. Brand, J. I. Choi, M. Jain, J. Mehlman, S. Katti, and P. Levis, "Applications of Self-Interference Cancellation in 5G and Beyond," IEEE Communications Magazine, vol. 52, no. 2, pp. 114-121, February 2014.

[8] M. Soumekh, Synthetic Aperture Radar Signal Processing. New York: Wiley, 1999.

[9] I. S. Merrill, Radar Handbook. NewYork: McGraw Hill, 1990, ch. 21.4. 